\title{
La réhabilitation prothétique après glossectomie partielle
}

\section{Prosthodontic rehabilitation after partiel glossectomy}

\section{MOTS-CLEFS :}

- Cancer de la langue, glossectomie radiothérapie, prothèse

\section{KEYWORDS}

- Tongue cancer, glossectomy, radiotherapy, prosthesis

$\operatorname{AOS} n^{\circ} 286-2017$

\section{Résumé}

Les glossectomies gardent encore une place prépondérante dans la stratégie thérapeutique des cancers linguaux. Elles peuvent avoir, selon leur siège et leur étendue, des conséquences plus au moins néfastes sur l'ensemble des fonctions orofaciales affectant ainsi la qualité de vie des patients.

Ce travail expose les différentes étapes de réalisation d'une prothèse adjointe complète chez un patient ayant subi un glossectomie partielle, suivie d'une radiothérapie pour traiter une tumeur maligne de la langue mobile. La confection prothétique avait pour objectif de rétablir les différentes fonctions de mastication, de phonation et de déglutition, d'améliorer l'esthétique et de réduire les séquelles émotionnelles et relationnelles du patient.
Abstract

The glossectomy still retain an important place in the therapeutic strategy of tongue cancers. According to its location and extent, it can have noxious disabilities of the entire orofacial functions and affects patients quality of life.

This work sets out the various steps of making a complete denture for a patient who underwent a partial glossectomy, followed by radiation therapy to treat malignant tumors of the oral tongue. The prosthetic rehabilitation aimed to restore mastication, phonation and swallowing, improve esthetics and reduce the emotional consequences of the patient.

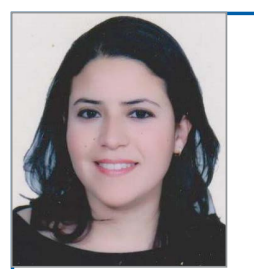

- Hasnaa ROKHSSI, Chirurgien dentiste, résidente en prothèse maxillo-faciale - Université Mohamed V, Faculté de médecine dentaire - Avenue Allal-El-Fassi, Rue Mohammed-Jazouli, Madinat Al Irfane, BP 6212, Rabat-Instituts, Maroc, hasnaa_ro@hotmail.com - rokhssi.hasnaa6@gmail.com - Tel : 00212667369776.

Mohammed AZHARI, Chirurgien dentiste, résident en prothèse maxillo-faciale - Université Mohamed V, Faculté de médecine dentaire - Avenue Allal-El-Fassi, Rue Mohammed-Jazouli, Madinat Al Irfane, BP 6212, Rabat-Instituts, Maroc.

Faiza BENFDIL, Professeur de l'enseignement supérieur en prothèse adjointe - Chef de département de prothèse adjointe - Université Mohamed $V$ - Faculté de médecine dentaire - Avenue Allal-El-Fassi, Rue Mohammed-Jazouli, Madinat Al Irfane, BP 6212, Rabat-Instituts, Maroc.

Nadia MERZOUK, Professeur de l'enseignement supérieur en prothèse adjointeChef de service de Prothèse adjointe - Université Mohamed V - Faculté de médecine dentaire - Avenue Allal-El-Fassi, Rue Mohammed-Jazouli, Madinat Al Irfane, BP 6212, Rabat-Instituts, Maroc.

Oussama BENTAHAR, Professeur agrégé en prothèse maxillo-faciale - Université mohamed V, - Faculté de médecine dentaire - Avenue Allal-El-Fassi, Rue MohammedJazouli, Madinat Al Irfane, BP 6212, Rabat-Instituts, Maroc. 


\section{INTRODUCTION}

Par ses 17 muscles, la langue posséde une grande mobilité participant à la mastication, la déglutition, la succion et l'articulation des sons [1].

Les carcinomes épidermoides de la langue sont parmi les tumeurs malignes les plus fréquentes des voies aérodigestives supérieures. Lorsque le diagnostic est posé à un stade précoce, l'attitude thérapeutique comporte une glossectomie partielle complétée par une radiothérapie postopératoire à laquelle peut s'ajouter une chimiothérapie [2].

Compte tenu de l'extension de la tumeur, la glossectomie peut être associée à une pelvectomie, voire même à une pelvi-mandibulectomie.

Il est certain que l'amputation partielle de la langue entraine une altération des différentes fonctions auxquelles elle participe. Il n'en demeure pas moins que celle-ci conserve une fonction plus ou moins raisonnable comparativement aux glossectomies totales [3]. Ces altérations fonctionnelles sont en plus aggravées par les complications liées à la chirurgie, notamment la présence de brides cicatricielles, l'installation d'une fibrose postchirurgicale, la suppression des remparts alvéolaires, la disparition des éléments de soutien du plancher buccal et la limitation de la mobilité linguale [4].

Hormis les lourdes séquelles de la chirurgie d'exérèse, le traitement adjuvant par irradiation présente de nombreuses conséquences qui touchent tous les tissus : cutané, muqueux, glandulaire, musculaire et osseux. L'hyposialie, la vulnérabilité tissulaire, le risque permanent d'ostéoradionécrose, la limitation d'ouverture buccale sont autant d'éléments défavorables à l'intégration prothétique [5].

Sur le plan psychologique, l'atteinte de l'intégrité physique ainsi que les conséquences fonctionnelles, en particulier l'altération de la phonation, sont souvent à l'origine d'un isolement du patient de son environnement familial et social.

Devant ces altérations, ces complications et ces séquelles qui font suite à la glossectomie, une intervention thérapeutique devient plus qu'impérative. Elle fait appel soit à une reconstruction chirurgicale, soit à une réhabilitation prothétique.

Dans le premier cas, de multiples contraintes telles que l'altération de l'état général, les répercussions d'une radiothérapie ultérieure, la difficulté de restaurer un organe lingual mobile et fonctionnel et la lourdeur de l'intervention, obligent parfois le patient et l'équipe médicale à surseoir à l'intervention chirurgicale. La réhabilitation prothétique s'impose alors de fait. Elle constitue une alternative simple et satisfaisante permettant d'améliorer la qualité de vie des patients et impose rigueur, précision et réflexion.
Selon le siège et le volume lingual réséqué, l'attitude prothétique est variable. Il s'agit en fait d'une réhabilitation individualisée. Dans le cas de glossectomie partielle, La réhabilitation privilégie une approche prothétique maxillaire [6]. Pour faciliter le contact entre le palais et le moignon lingual résiduel, un abaissement dit aussi une augmentation de la voûte palatine prothétique est réalisé $[6,7,8,9,10,11,12]$. En présence d'une large résection linguale avec limitation importante de sa mobilité, une augmentation linguale est souvent envisagée [12].

Dans le cas de glossectomie totale ou subtotale, l'approche est différente. Elle fait appel à des prothèses mandibulaires présentant des extensions sur lesquelles viennent se fixer une prothèse linguale en résine ou en silicone $[6,8,11,13,14]$.

Le cas présenté a pour objectif d'illustrer les différentes étapes d'élaboration d'une prothèse complète bimaxillaire chez un homme de 60 ans présentant une perte de substance partielle de la langue séquellaire au traitement chirurgical, et de mettre en relief l'intérêt de la création d'une anatomie fonctionnelle de l'extrados prothétique par une augmentation palatine et linguale dans la gestion des troubles fonctionnels, esthétiques et psychologique qui en découlent.

\section{PRÉSENTATION DU CAS CLINIQUE}

Un patient âgé de 60 ans, édenté complet, a consulté pour une réhabilitation prothétique.

L'interrogatoire a révélé :

$\checkmark$ des antécédents de chirurgie d'exérèse d'un carcinome épidermoide de la partie marginale gauche de la langue mobile suivie d'une radiothérapie postopératoire de 70 grays ;

$\checkmark$ des difficultés d'élocution avec des paroles incompréhensibles réduisant ses capacités de communication avec l'entourage par perte des appuis et réduction de la mobilité du massif lingual résiduel ;

$\checkmark$ une gêne à la déglutition par modification des appuis linguaux, présence de brides cicatricielles et glossopexie ; $\checkmark$ une perturbation de l'alimentation du fait de l'absence de toute prothèse, des difficultés de manipulation et du maintien du bol alimentaire entre les arcades dentaires et de la stagnation alimentaire en regard de la perte de substance.

\section{L'EXAMEN EXOBUCCAL}

Cet examen a mis en évidence un affaissement de l'étage inférieur (Fig. 1), une dépilation cutanée 


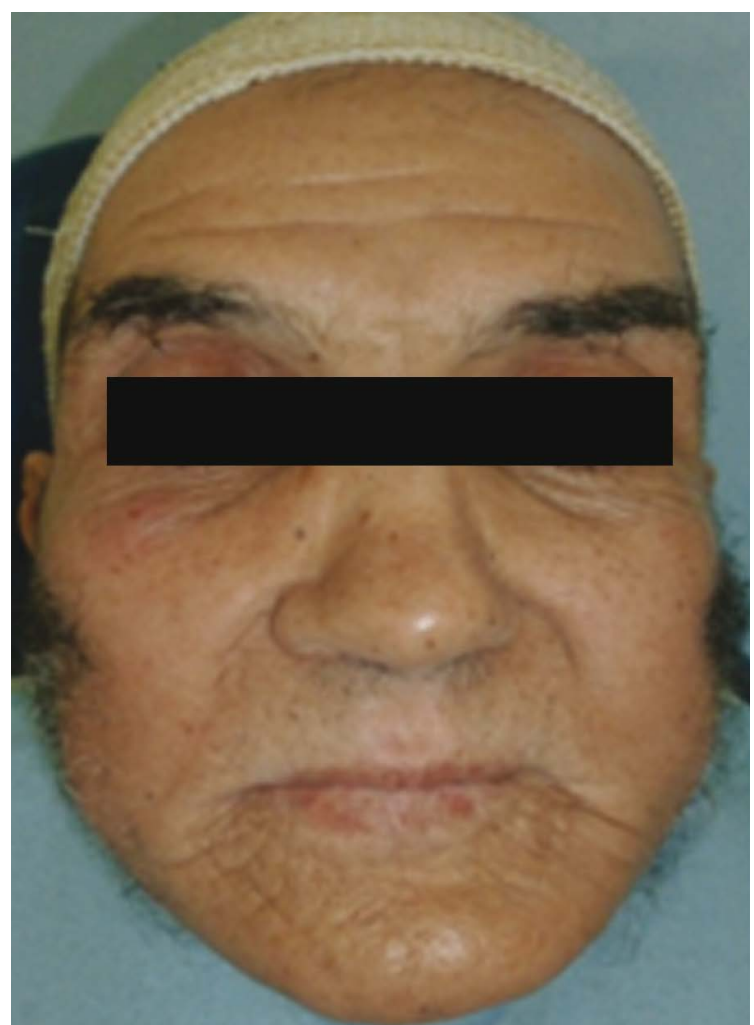

$\triangle$ Fig. 1 : Étage inférieur réduit en rapport avec l'édentation.

particulièrement visible en regard des champs d'irradiation (Fig. 2) ainsi qu'une limitation de l'ouverture buccale. Les lèvres sont sèches et présentent une perlèche commissurale (Fig. 3).

\section{L'EXAMEN ENDOBUCCAL}

L'examen endobuccal a montré :

I un édentement complet bimaxillaire ;

I une glossectomie partielle intéressant la partie marginale gauche de la langue mobile avec une réduction de la mobilité du moignon lingual résiduel (Fig. 4); $\checkmark$ une vacuité résiduelle siégeant entre la langue et la table interne de la crête alvéolaire mandibulaire favorisant la rétention alimentaire et allongeant le temps consacré aux repas (Fig. 5) ;

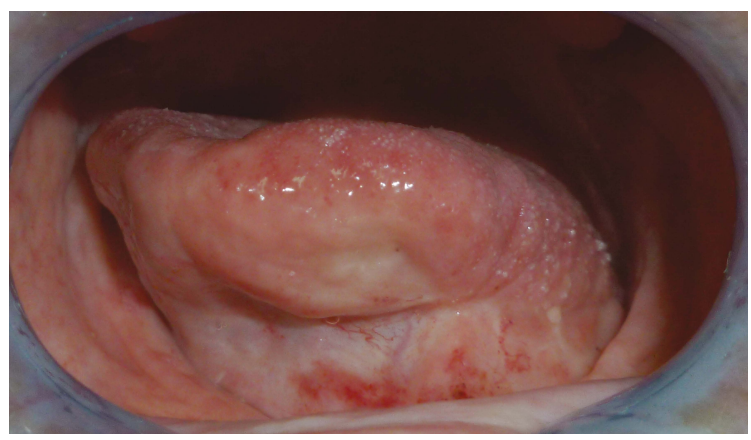

$\triangle$ Fig. 4 : Glossectomie partielle gauche et limitation de la mobilité linguale.

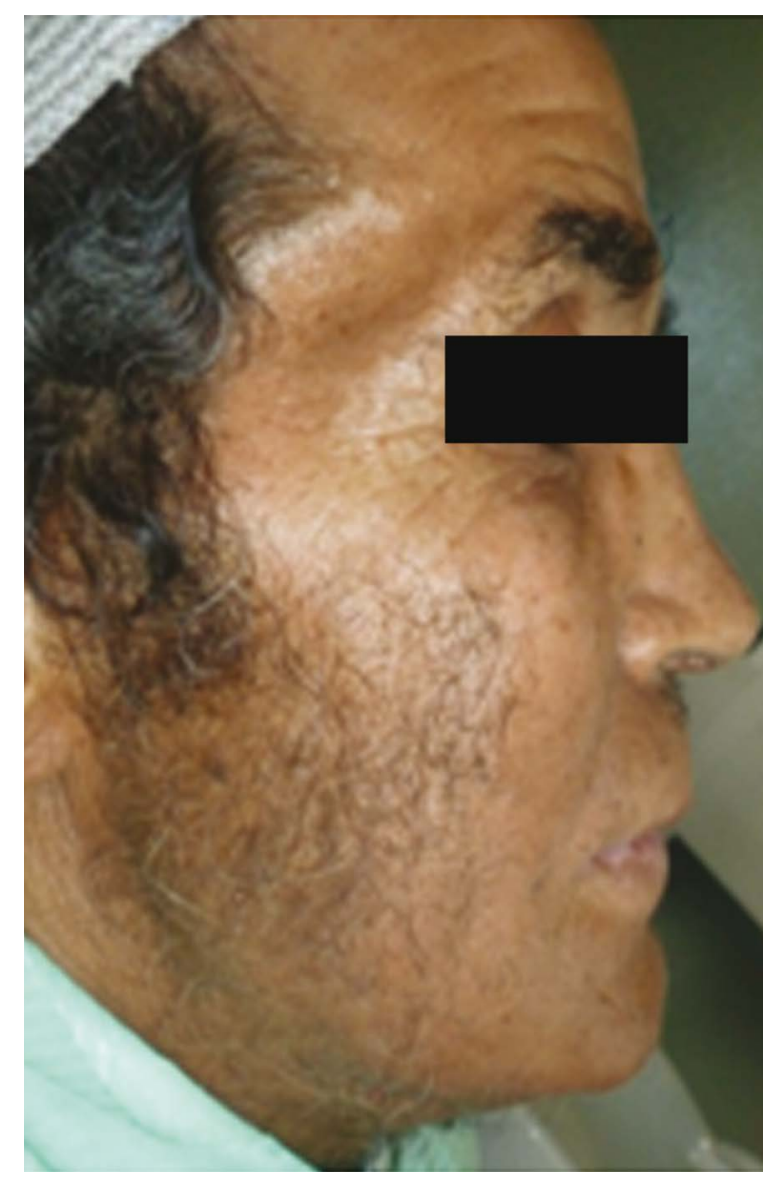

$\triangle$ Fig. 2 : Dépilation cutanée en regard des champs d'irradiation.

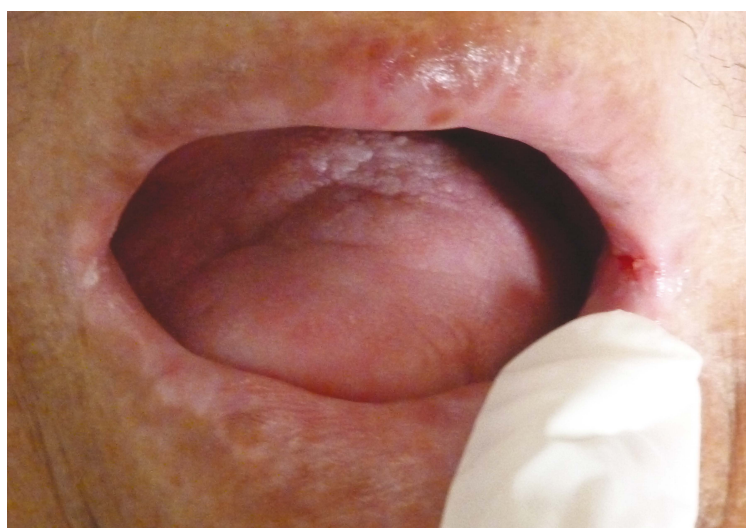

$\triangle$ Fig. 3 : Lèvres sèches avec une perlèche commissurale.

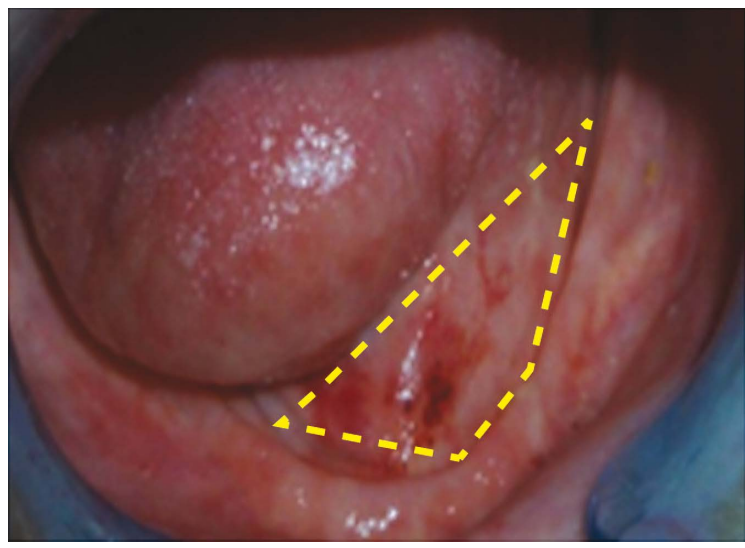

$\triangle$ Fig. 5 : Vacuité siégeant entre la langue et la table interne de la crête alvéolaire. 
une crête mandibulaire sans relief rendant difficile la rétention et la stabilisation prothétique (Fig. 5); $\checkmark$ une muqueuse buccale sèche, fine et fragile recouverte d'une salive épaisse et collante au miroir (Fig. 6).

\section{OBJECTIFS THÉRAPEUTIQUES}

L'état actuel des connaissances, montre qu'il est impossible de restaurer la fonction originelle de la langue tant chirurgicalement que prophétiquement. Il est cependant possible de rétablir partiellement la fonction et l'esthétique à l'aide d'une prothèse. La réhabilitation prothétique a pour objectif de $[6,11]$ : 1. réduire la taille de la cavité orale de manière à améliorer les caractéristiques de la cavité de résonnance ;

2. diriger le bol alimentaire vers l'oropharynx ;

3. protéger les tissus sous-jacents ;

4. augmenter la surface résiduelle linguale en contact avec le palais lors de la phonation et de la déglutition ; 5. améliorer l'esthétique et réduire le préjudice psychologique.

\section{DÉCISION THÉRAPEUTIQUE ET PLAN DE TRAITEMENT}

Une prothèse complète mandibulaire stabilisée par deux implants a été proposée au patient comme traitement de choix. Cependant, pour éviter d'autres interventions chirurgicales, le patient a opté pour une prothèse complète conventionnelle. La réalisation prothétique est faite en plusieurs temps successifs : tout d'abord, des prothèses amovibles complètes sont réalisées de façon classique. Lors des séances de contrôle, le patient était insatisfait en raison de la gêne persistante pendant la phonation et la déglutition et de la stagnation des aliments en regard de la perte de substance linguale. Dans un deuxième temps et afin de faciliter le contact entre la langue et le palais, une augmentation palatine est réalisée. Par la suite une

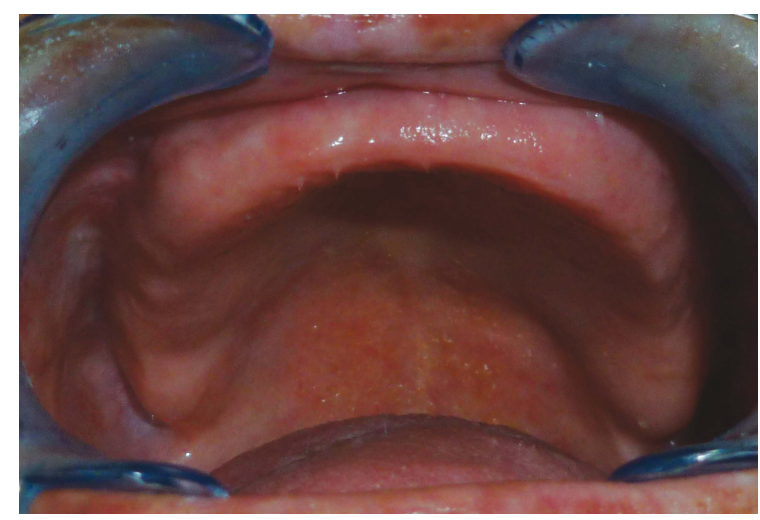

$\triangle$ Fig. 6 : Muqueuse irradiée sèche, mince et dépolie.

augmentation linguale est effectuée pour améliorer la stabilité et le confort du patient.

\section{DÉMARCHE THÉRAPEUTIQUE}

\section{Réalisation d'une prothèse complète bimaxillaire classique (Fig. 7)}

Les étapes classiques de la prothèse adjointe complète sont réalisées en respectant les particularités liées au terrain irradié.

Le patient irradié est un sujet à risque. La réhabilitation prothétique doit prendre en considération la vulnérabilité tissulaire ainsi que le risque permanent de survenue d'ostéoradionécrose. De ce fait, elle doit être atraumatique durant les différentes séquences thérapeutiques $[5,15]$ :

$\checkmark$ les empreintes ne doivent pas être compressives, le plâtre, la pâte de Kerr et l'oxyde de zinc eugénol sont à éviter. Des matériaux élastiques tels que les polyéthers et les polysulfures sont les plus adaptés ;

$\checkmark$ en raison du trismus, la dimension verticale d'occlusion est légèrement réduite pour faciliter l'insertion prothétique et la mastication ;

$\checkmark$ les dents sont en résine car elles absorbent mieux les chocs et sont donc moins traumatisantes pour les muqueuses en comparaison avec les dents en céramique ;
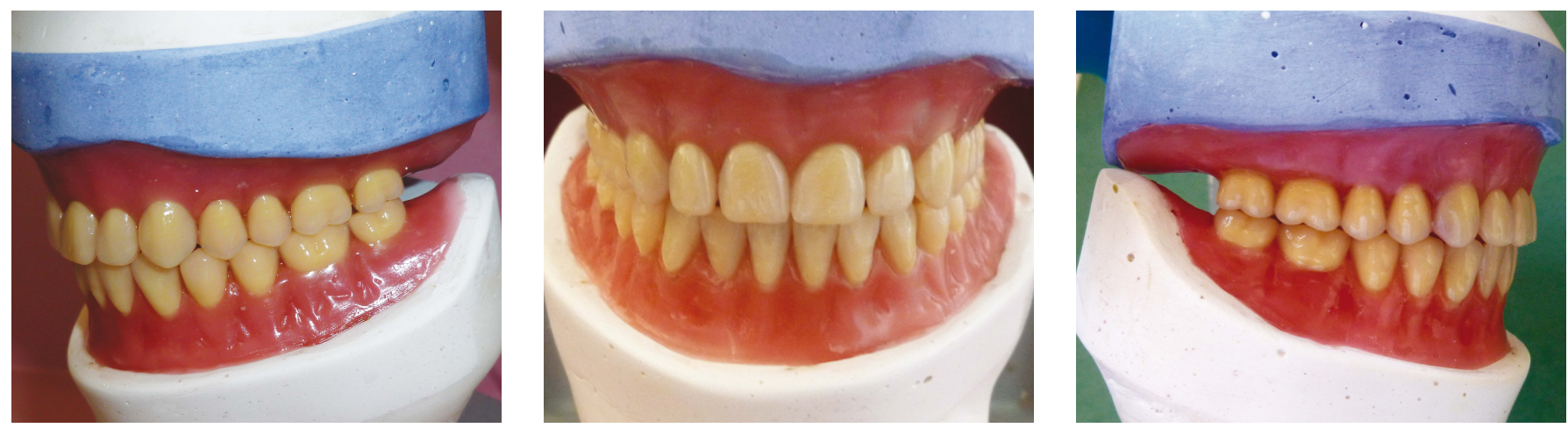

$\triangle$ Fig. 7 : Prothèses mandibulaire et maxillaire produites de façon classique pour remplacer les dents manquantes. 
lors de la pose, toute compression ou surextension est minutieusement éliminée ;

$\checkmark$ l'équilibration occlusale est conduite de façon rigoureuse afin d'éviter toute surcharge occlusale ;

$\checkmark$ les bords et l'intrados de la prothèse doivent être lisses ;

I un contrôle rigoureux est programmé systématiquement avec des séances rapprochées ;

I des substituts salivaires sont prescrits pour réduire la xérostomie.

\section{Modification de la prothèse supérieure par augmentation palatine} (Fig. 8, 9, 10)

Afin de permettre une restauration des fonctions buccales auxquelles participe la langue, il est nécessaire de recréer un contact du moignon lingual avec le palais ainsi qu'avec les dents prothétiques antérieures.

Ce contact est permis par un abaissement de la voûte palatine obtenue par une empreinte fonctionnelle enregistrant les mouvements du moignon lingual résiduel lors des différentes fonctions orales. Cette empreinte est réalisée en rebasant par la mise en place d'une résine a prise retardée en phase plastique au niveau de l'extrados palatin (Fig 8a). La prothèse est portée par le patient pendent 3 jours, ce qui constitue une empreinte fonctionnelle ambulatoire du moignon lingual. Celle-ci est ensuite affinée par un surfaçage au polysulfure light après application d'un adhésif univesel (Fig. 8b et c).

La transformation en résine acrylique est effectuée après la réalisation d'une clé en plâtre (Fig. 9, 10).

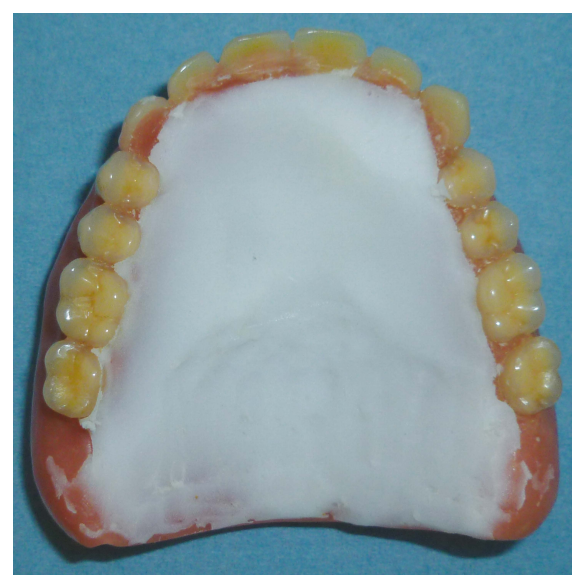

$\triangle$ Fig. 8a : Empreinte fonctionnelle de l'extrados prothétique traduisant les contacts palatins de la langue lors de la phonation et de la déglutition avec une résine à prise retardée.

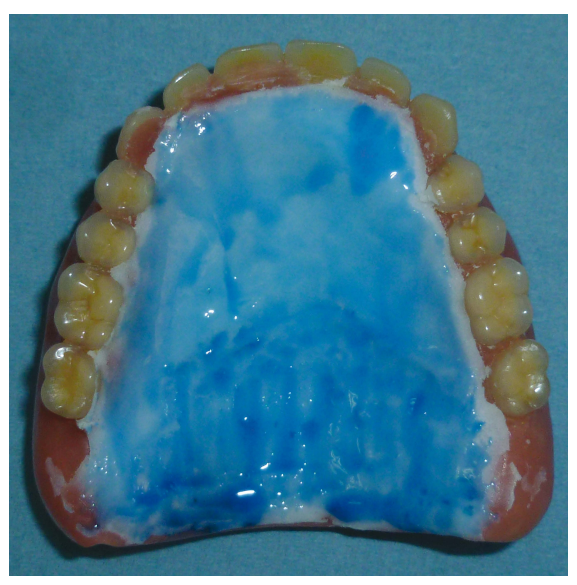

$\triangle$ Fig. 8b : Application de l'adhésif universel.

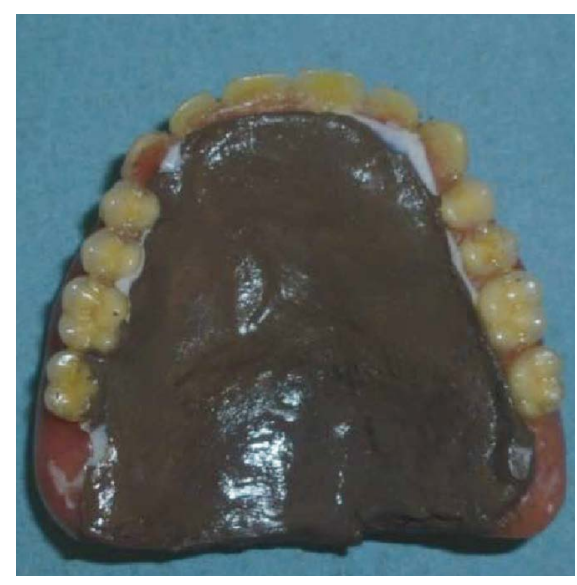

$\triangle$ Fig. 8c : Lavis avec un polysulfure de basse viscosité.

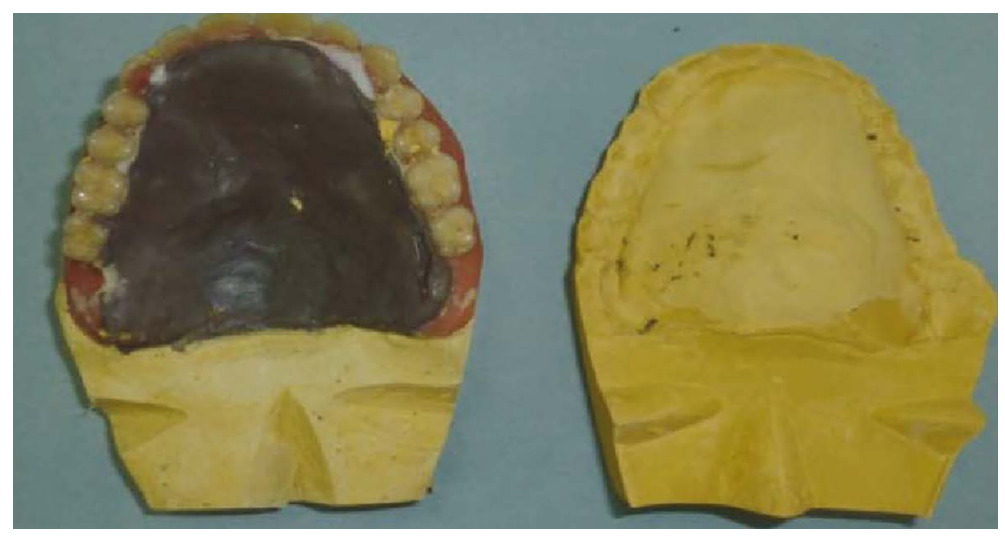

$\triangle$ Fig. 9 : Rajout de la résine acrylique est effectué après la réalisation d'une clé en plâtre.

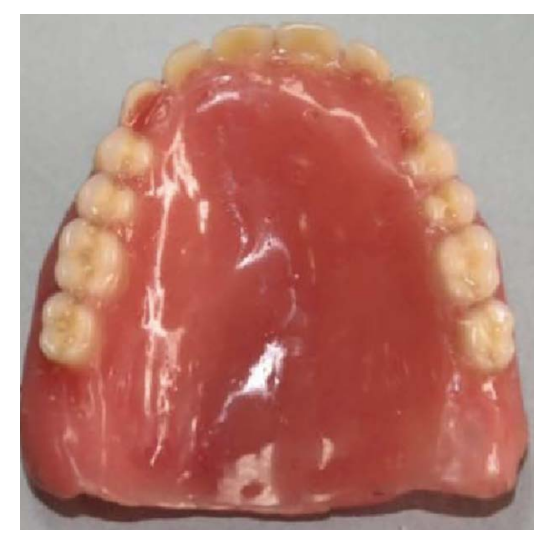

$\triangle$ Fig. 10 : Augmentation de la voûte palatine : extrados prothétiques après polymérisation. 
Modification de la prothèse mandibulaire (Fig. 11a, 11b, 11c, 12, 13)

Afin d'éviter la rétention alimentaire en regard de la perte de substance linguale, et d'améliorer la stabilité prothétique et le confort du patient, la prothèse mandibulaire est rebasée au Fitt ${ }^{\star}$ de Kerr pour enregistrer les surfaces polies stabilisatrices en regard de la langue reséquée (Fig. 11a, b, c). La prothèse est ensuite enduite d'adhésif, séchée et garnie d'un polysulfure de basse viscosité (Fig. 12). Elle est introduite en bouche. Le patient est prié de fermer en occlusion centrée et d'effectuer tous les mouvements fonctionnels de la langue. Les surfaces polies stabilisatrices en regard du moignon réséqué sont ainsi enregistrées. La prothèse est ensuite envoyée au laboratoire pour rebasage (Fig. 13).
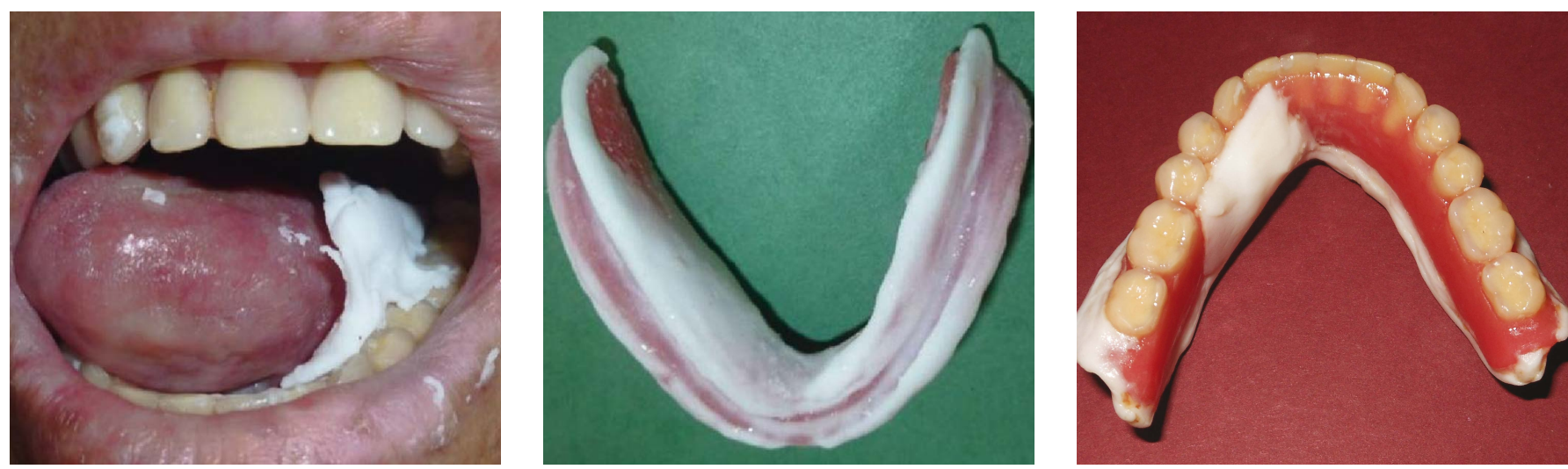

$\triangle$ Fig. 11 : Harmonisation du profil de la prothèse mandibulaire avec l'activité musculaire linguale après rebasage avec une résine à prise retardée.

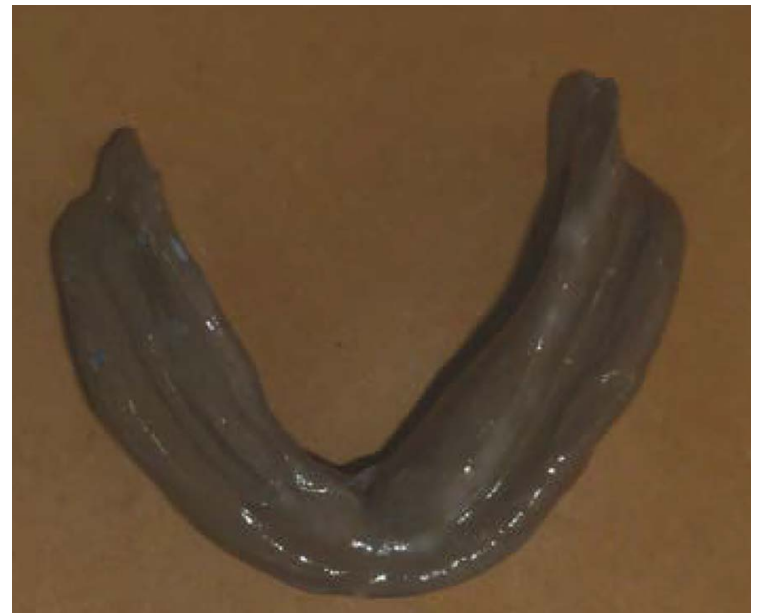

$\triangle$ Fig. 12 : Empreinte finale au polysulfure light.

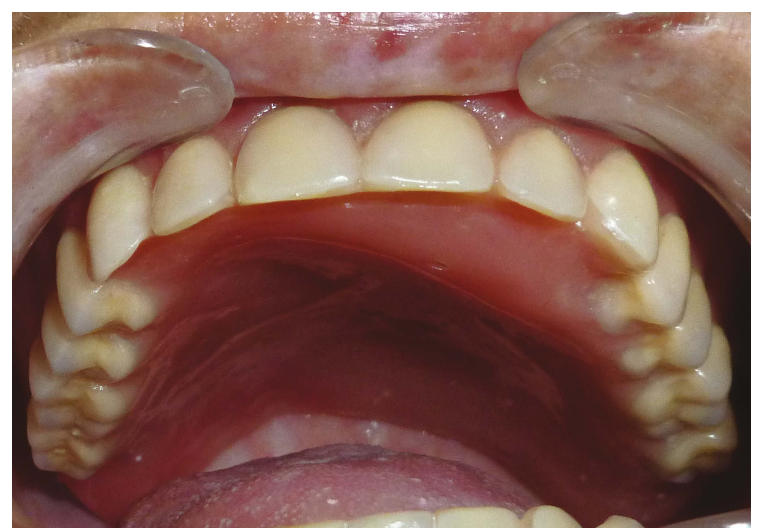

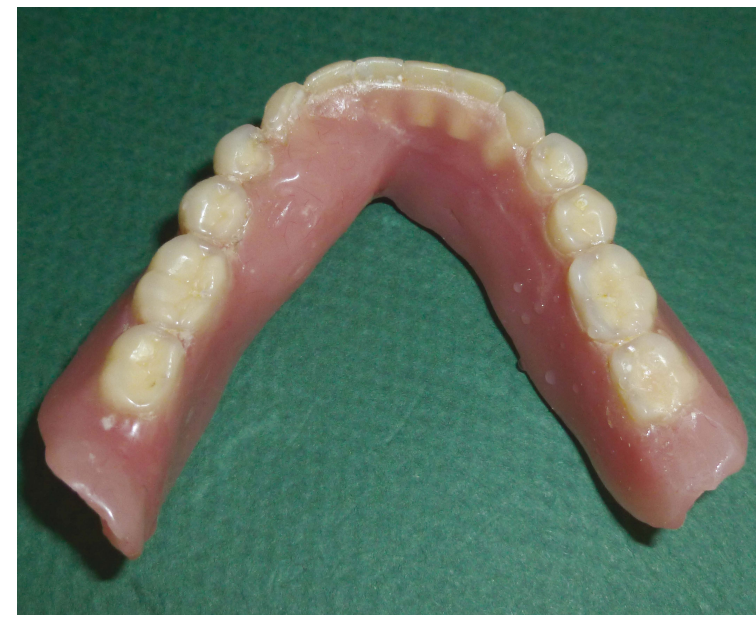

$\triangle$ Fig. 13 : Augmentation linguale.

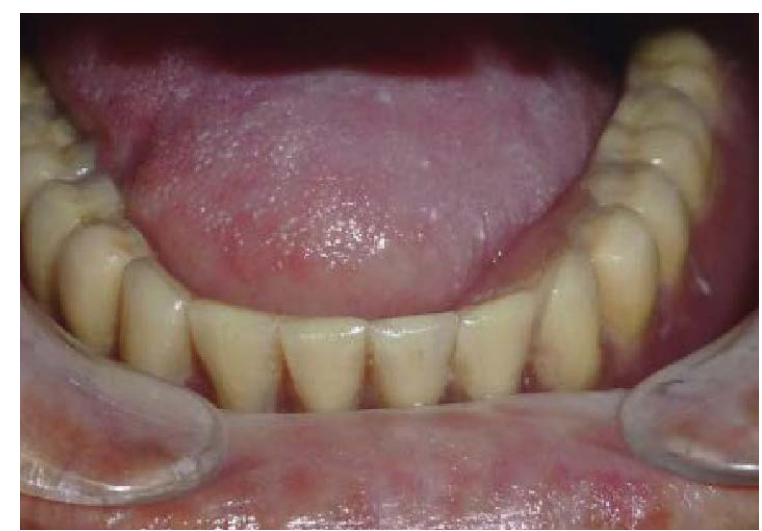

$\triangle$ Fig. 14 : Prothèses finales : augmentation maxillaire et mandibulaire. 
Insertion de la prothèse et maintenance (Fig. 14a et b, Fig. 15)

Une précaution particulière a été portée à l'élimination de toute zone de compression et au polissage soigneux des prothèses pour éviter tout traumatisme et irritation muqueuse (Fig. 14a, Fig. 14b).

Des contrôles réguliers toutes les semaines sont effectués, les prothèses ont été retouchées légèrement au niveau de l'extrados prothétique en rapport avec la résection linguale afin d'améliorer le confort du patient. Lors des visites de contrôle, le patient a rapporté une nette amélioration des différentes fonctions orales de mastication, de déglutition et de phonation. Il a témoigné sa satisfaction du résultat obtenu tant sur les plans esthétique, fonctionnel que sur les plans stabilitérétention prothétiques, par son large sourire (Fig. 15).

\section{CONCLUSION}

Les glossectomies non reconstruites chirurgicalement laissent de lourdes séquelles fonctionnelles, esthétiques et psychologiques.

Les prothèses augmentatrices de palais ou les prothèses linguales, surtout en présence d'un moignon lingual

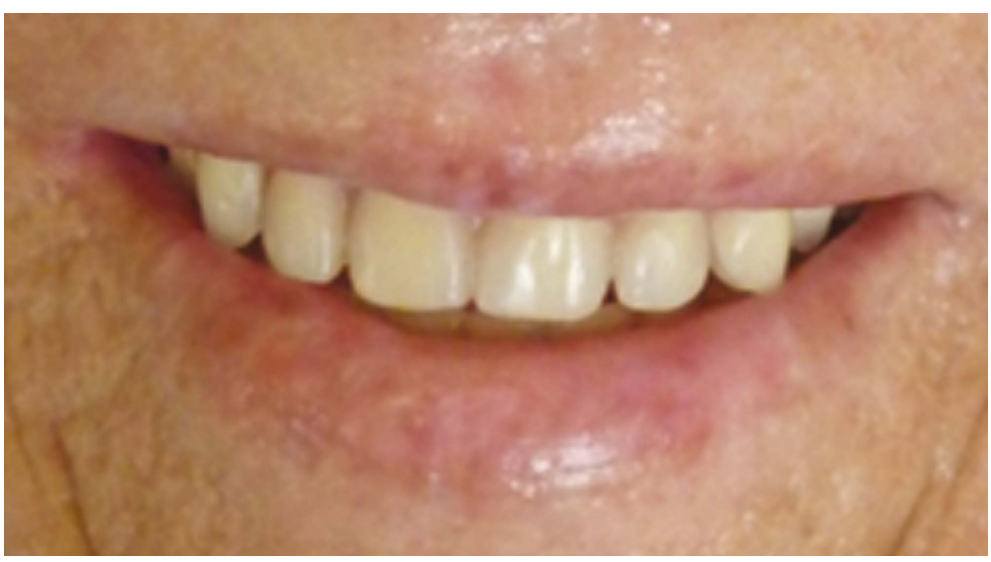

$\triangle$ Fig. 15 : Sourire de satisfaction du patient.

mobile, permettent d'aider le patient à retrouver des fonctions phonatoires et de déglutition proches de la normalité et ainsi d'améliorer la qualité de vie des patients.

Il faut garder à l'esprit que chaque cas est singulier et que le praticien doit s'adapter à chaque situation clinique, en adoptant le plus souvent la solution la plus simple.

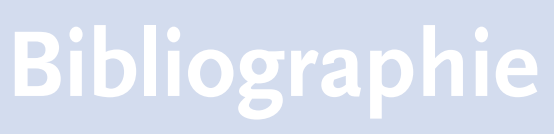

[1] Dassonville O, Poissonnet G, Bozec A. Glossectomies. Encyclopédie MédicoChirurgicale, Tête et Cou. 46-250, 2006.

[2] Prades J-M, Schmitt T, Timoshenko A. Cancer de la langue. Encyclopédie MédicoChirurgicale Oto-rhino-laryngologie, 20-627-A-10, 2003.

[3] Lefevre C et al. Restauration prothétique après glossectomie totale. Stratégie Prothétique 2003; 3: 117-123.

[4] Beumer III J, Marunick M-T, Silverman S. Rehabilitation of tongue and mandibular defects. In: Maxillofacial rehabilitation, prosthodontic and surgical management of cancer-related, acquired, and congenital defects of the head and neck, 2011, Beumer III J, Marunick Mark T, Esposito S-J, Maryland University edit, $3^{\text {rd }}$ ed. 213-254.

[5] Fajri L, Benfdil F, Bentahar O, El Mohtarim B, Abdedine A. Radiothérapie et restauration prothétique de l'édenté complet. Clinic 2008; 29: 81-87.

[6] Fusaro S, Destruhaut F, Vigarios E, Toulouse E, Pomar P. Réhabilitation prothétique après glossectomie. Encyclopédie Médico-Chirurgicale Stomatologie, 22-066-B-54, 2008, Médecine buccale, 28-565-G-10, 2009.

[7] Marunick M, Tselios N. The efficacy of palatal augmentation prostheses for speech and swallowing in patients undergoing glossectomy: a review of the literature. J Prosthet Dent 2004; 91(1): 67-74.

[8] Frank RL, Vergo Th, Schaaf NG, Zimmerman R. Prosthodontic and speech rehabilitation after partiel and complete glossectomy. J Prosthet Dent 1980; 43(2): 204-211.

[9] Gopi Maj A, Dhiman Col R.K, Kumar Col D. Customizing the palatal contour of a complete denture using palatogram in a case of partiel glossectomy. Medical J Armed Forces India 2013: 1-4.

[10] McKinstry R, Armany M A, Beery Q, Sansone F. Speech considerations in prosthodontic rehabilitation of the glossectomic patient. J Prosthet Dent 1985; 5(3): 385-387.

[11] Armany M, Downs JA, Beery Q, Aslan Y. Prosthodontic rehabilitation of glossectomy patients. J Prosthet Dent 1982; 48(1): 78-81.

[12] Okuno K, Nohara K, Tanaka N, Sasao Y, Sakai T. The efficacy of a lingual augmentation prosthesis for swallowing after a glossectomy: a clinical report. J Prosthet Dent 2014; 111: 342-345.

[13] Clercq J. La réhabilitation prothétique du patient glossectomisé : un cas clinique. J Ordre Dentistes Quebec 2011; 48(1): 7-13.

[14] Pigno M, Funk J. Prosthetic management of a total glossectomy defect after free flap reconstruction in an edentulous patient: a clinical report. J Prosthet Dent 2003; 88: 119-122.

[15] Merzouk N, Berrada S, Benfdil F, Abdedine A. Les critères de choix des matériaux et des techniques d'empreinte en prothèse amovible partielle. Actual Odonto-Stomatol 2008; 243: 265-78. 